\title{
ANÁLISIS DE LAS COMPETENCIAS Y CONOCIMIENTOS DE LOS ESTUDIANTES EMPRESARIALES MEDIANTE EL EMPLEO DE LA ESTRATEGIA ACTIVA DE APRENDIZAJE "MAPAS CONCEPTUALES"
}

Analysis of the competences and knowledge of business students through the use of the active learning strategy "conceptual maps"

Análise das competências e conhecimentos de estudantes de negócios, através do uso da estratégia de aprendizagem ativa "mapas concetuais"

\section{Leyla Angélica Sandoval Hamón (1)}

(1) Universidad Autónoma de Madrid, España. Teléfono: +34 914975518. Correo electrónico: angelica.sandoval@,uam.es

\section{Resumen}

En el Espacio Educación Superior Europea (EEES), los estudios de titulación oficial deben fomentar las competencias y el conocimiento de los estudiantes. Esta experiencia se basa en la estrategia activa de aprendizaje (mapas conceptuales) donde los estudiantes analizan, sintetizan y resumen los temas propuestos en clase, con el fin de mejorar sus conocimientos y habilidades. Por lo tanto, este trabajo describe la experiencia de aplicar la estrategia "mapas conceptuales" en el tema de "Fundamentos de la Administración de Empresas" en el primer semestre del año académico 20182019. La implementación de esta experiencia requirió una revisión de los temas, su seguimiento y evaluación. Las conclusiones (derivadas del análisis de datos de cuestionarios de competencia y pruebas de conocimiento) indican que este tipo de estrategia aumenta la comprensión de los conceptos básicos que se enseñan sobre la materia mencionada anteriormente, pero no aumenta la competencia de los estudiantes sobre la materia.

Palabras clave: Universidad; mapas conceptuales; conocimientos; competencias

\section{Abstract}

In the European Higher Education Area (EHEA) official degree studies must foster competences as well as knowledge in students. This experience is based on the active 
learning strategy (conceptual maps) where students analyze, synthesize and outline topics proposed in class, in order to improve their knowledge and skills. Therefore, this work describes the experience of applying the strategy "conceptual maps" on the subject of "Fundamentals of Business Administration" in the first semester of the 2018-2019 academic year. The implementation of this experience required a review of the topics, their follow-up and evaluation. The conclusions (derived from the analysis of data from competency questionnaires and knowledge tests) indicate that this type of strategy increases the understanding of the basic concepts taught on the aforementioned subject but does not increase the students' competence on the subject.

Keywords: University; conceptual maps; knowledges; competences

\section{Resumo}

No Espaço Europeu de Ensino Superior (EEES), os estudos para a graduação devem fomentar as competências e o conhecimento dos alunos. Esta experiência baseia-se na estratégia de aprendizagem ativa (mapas concetuais), onde os alunos analisam, sintetizam e resumem os temas propostos na aula, a fim de melhorar seus conhecimentos e habilidades. Neste sentido, este trabalho descreve a experiência de aplicar a estratégia "mapas conceituais" na disciplina "Fundamentos de Administração de Empresas" no primeiro semestre do ano letivo 2018-2019. A implementação desta experiência exigiu uma revisão dos temas, o seu acompanhamento e avaliação. As conclusões (derivadas da análise de dados de questionários de competência e testes de conhecimento) indicam que este tipo de estratégia aumenta a compreensão dos conceitos básicos ensinados sobre a matéria anteriormente mencionada, mas não aumenta a competência dos alunos sobre a mesma.

Palavras-chave: Universidade; mapas concetuais; conhecimento; competências

\section{Introducción}

En el Espacio Europeo de Educación Superior (EEES) los estudios de titulación oficial deben fomentar las competencias y el conocimiento de los estudiantes. Tomando como referencia esta premisa, en los últimos años se han venido presentando incesantes cambios que abarcan planes de estudio, estrategias, evaluaciones, tecnologías, etc. 
El empleo de estrategias y metodologías alternativas a las clases magistrales ha proliferado en la mayoría de los planes de estudios como un medio de orientar a los estudiantes a explorar nuevas vías de construcción de su conocimiento para favorecer que adquieran otras competencias. Una definición que se ha tenido como referencia de metodologías activas es que "el aprendizaje activo implica proporcionar oportunidades a los estudiantes para hablar y escuchar de manera significativa, escribir, leer, y reflexionar sobre los contenidos, ideas, problemas y preocupaciones de una materia académica" (Meyers y Jones, 1993, p. 6). Así, algunas estrategias activas de aprendizaje según Zayapragassarazan y Kumar (2012) son: mapas conceptuales, redacción colaborativa, panel de discusión, etc.

El reto de emplear estrategias alternativas en la Educación Superior ha generado incógnitas sobre su eficacia. Esto ha llevado a que docentes reflexionen sobre el impacto real que tienen estas nuevas metodologías y estrategias activas.

En relación con estos precedentes, el objetivo de este trabajo es analizar empíricamente (es decir, buscar evidencias con los datos) el rendimiento académico y el desarrollo de competencias de los estudiantes de Ciencias Empresariales al emplearse la estrategia activa: mapas conceptuales (donde se ilustra en este caso, las relaciones entre ideas a partir de los temas sugeridos en la asignatura).

\section{Contextualización}

La experiencia se ha analizado en la asignatura de "Fundamentos de Administración y Dirección de Empresas" (FADE) (6 créditos) que se imparte en el primer año del grado de Administración y Dirección de Empresas en la Universidad Autónoma de Madrid. El primer semestre del curso 2018-2019 fue el periodo en que se realizó esta experiencia y el número total de estudiantes fue de 50 .

En la asignatura FADE, se pretende que el estudiante conozca los conceptos básicos relativos a la economía de la empresa y el papel que las organizaciones empresariales.

El equipo que supervisó la experiencia está conformado por tres docentes del departamento de organización de empresas (dos de ellos responsables directos de la asignatura) y un profesor del departamento psicología y metodología. Los docentes han replanteado la forma de impartir la asignatura tomando como referencia los mapas conceptuales. 


\section{Diseño y desarrollo}

Fase I: Cuestionario inicial (Pre-test) y final (Post-test) de competencias y conocimientos en la asignatura. Este cuestionario se adaptó tomando como referencia el propuesto por Palacios Picos, López-Pastor y Fraile Aranda (2018). La adaptación del cuestionario fue validada a través de una prueba piloto con 5 estudiantes del grado de Administración de Empresas de la UAM. Una vez aplicado el cuestionario a los estudiantes, los datos se analizaron con modelos mixtos (software SPSS v20).

Fase II: -Diseño y ejecución de mapas conceptuales. Donde se analizaron temas como "Empresario, estructura de la propiedad y control; El mercado y el entorno". Los estudiantes debían analizar la información y realizar los pertinentes esquemas a través de software como CmapTools. El proceso de elaboración y presentación de estos mapas conceptuales tuvo como referencia una rúbrica.

\section{Evaluación}

Los resultados derivados de la aplicación del cuestionario sobre la evaluación de competencias inicial (Pre-test) y final (Post-test) teniendo en cuenta la iniciativa de mapas conceptuales se presentan en la tabla 1, y en las figura 1 y 2 se observan sus respectivas representaciones gráficas. Estos resultados indican que el grupo 115 obtuvo unas puntuaciones en competencias más elevadas al aplicarse el primer cuestionario que el segundo; así, según lo estudiantes, la estrategia activa empleada no mejoró sus competencias. En cuanto a los resultados de las pruebas de conocimientos presentadas en la tabla 2, representadas en las figuras 3 y 4 , se deduce que los estudiantes reconocen que la estrategia aumentó su rendimiento académico.

Tabla 1.

Evaluación de competencias

\begin{tabular}{|c|c|c|c|}
\hline \multirow{2}{*}{\multicolumn{2}{|c|}{ Estadísticos }} & \multicolumn{2}{|c|}{ Grupo 115} \\
\hline & & Pre-test & Post-test \\
\hline \multicolumn{2}{|c|}{$\mathrm{N}$} & 47 & 20 \\
\hline \multicolumn{2}{|l|}{ Media } & 17,34 & 15,80 \\
\hline \multicolumn{2}{|l|}{ Mediana } & 17,00 & 16,00 \\
\hline \multicolumn{2}{|l|}{ Desv. típ. } & 3,299 & 3,054 \\
\hline \multicolumn{2}{|c|}{ Asimetría } & 0,433 &,- 258 \\
\hline \multicolumn{2}{|c|}{ Error típ. de asimetría } & 0,347 & 0,512 \\
\hline \multicolumn{2}{|c|}{ Curtosis } & 0,882 & $-1,098$ \\
\hline \multicolumn{2}{|c|}{ Error típ. de curtosis } & 0,681 & 0,992 \\
\hline \multirow[t]{3}{*}{ Percentiles } & 25 & 16,00 & 13,00 \\
\hline & 50 & 17,00 & 16,00 \\
\hline & 75 & 19,00 & 19,00 \\
\hline
\end{tabular}

Análisis de las competencias y conocimientos de los estudiantes empresariales mediante el empleo de la estrategia activa de aprendizaje "mapas conceptuales" 


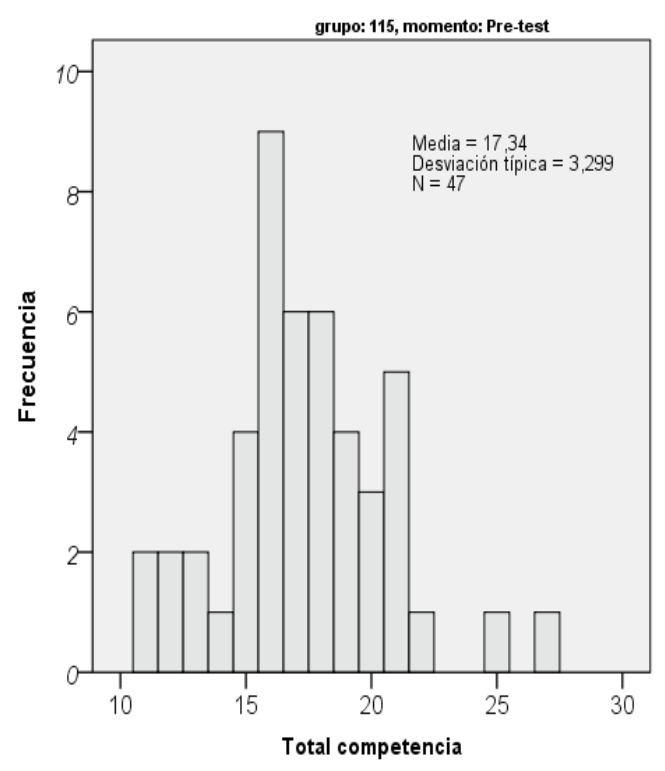

Figura 1. Evaluación de competencias inicial

Tabla 2.

Prueba de conocimientos - Número de aciertos

\begin{tabular}{|c|c|c|c|}
\hline \multirow{2}{*}{\multicolumn{2}{|c|}{ Estadísticos }} & \multicolumn{2}{|c|}{ Grupo 115} \\
\hline & & Pre-test & Post-test \\
\hline \multicolumn{2}{|c|}{$\mathrm{N}$} & 42 & 37 \\
\hline \multicolumn{2}{|l|}{ Media } & 5,76 & 6,95 \\
\hline \multicolumn{2}{|l|}{ Mediana } & 6,00 & 7,00 \\
\hline \multicolumn{2}{|l|}{ Desv. típ. } & 1,792 & 1,929 \\
\hline \multicolumn{2}{|l|}{ Asimetría } & $-0,42$ & $-0,88$ \\
\hline \multicolumn{2}{|c|}{ Error típ. de asimetría } & 0,37 & 0,39 \\
\hline \multicolumn{2}{|c|}{ Curtosis } & 0,91 & 0,61 \\
\hline \multicolumn{2}{|c|}{ Error típ. de curtosis } & 0,72 & 0,76 \\
\hline \multirow{3}{*}{ Percentiles } & 25 & 6 & 6 \\
\hline & 50 & 7 & 7 \\
\hline & 75 & 8 & 8 \\
\hline
\end{tabular}

Número de aciertos al examen
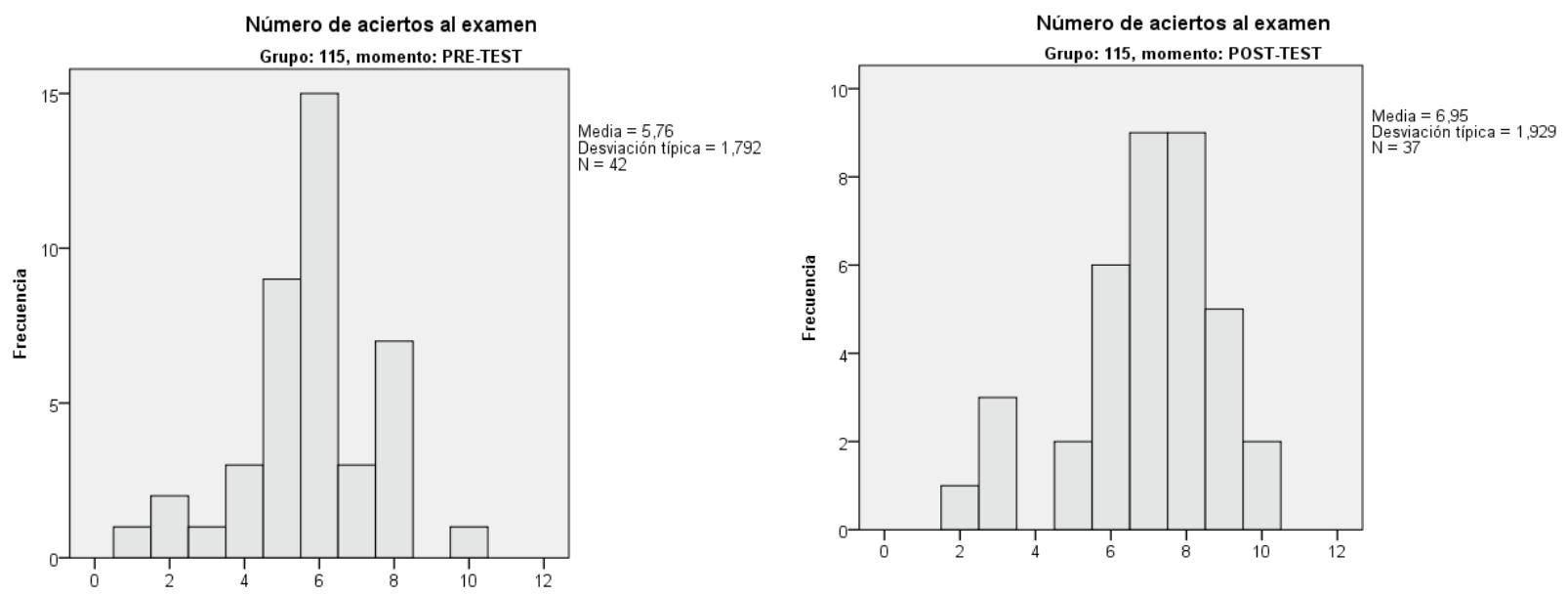

Figura 3. Distribución prueba de conocimientos incial

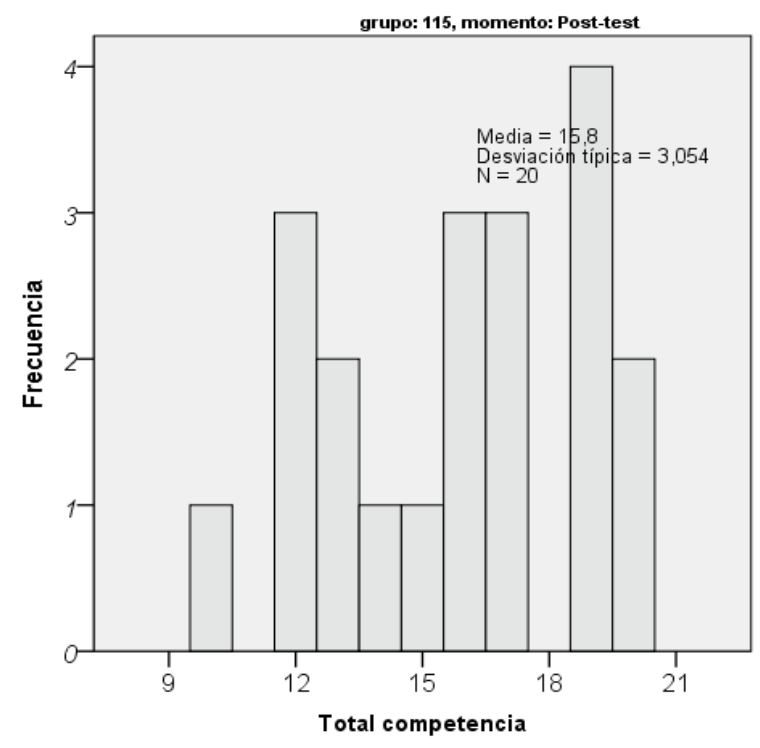

Figura 2. Evaluación de competencias final
Figura 4. Distribución prueba de conocimientos final

Análisis de las competencias y conocimientos de los estudiantes empresariales mediante el empleo de la estrategia activa de aprendizaje "mapas conceptuales" 


\section{Conclusiones}

En la evaluación de competencias y conocimientos de la asignatura FADE, tuvo como punto de referencia los mapas conceptuales (se dispuso una rubrica para su elaboración) y se realizaron dos pruebas de conocimientos.

En este marco, la experiencia buscó contribuir al proceso de construcción del aprendizaje por otra vía. Esta estrategia resultó provechosa para ordenar sus ideas y aclarar dudas de contenidos. Esto se corrobora con los resultados de las pruebas de conocimientos donde si aumentaron los niveles de rendimientos de los estudiantes. En el caso del cuestionario de competencias queda en evidencia la necesidad de proporcionar mayor a los estudiantes mayor formación en manejo de software (como Cmaptools).

\section{Referencias}

Meyers, C., \& Jones, T. B. (1993). Promoting Active Learning. Strategies for the College Classroom. Jossey-Bass Inc., Publishers, 350 Sansome Street, San Francisco, CA 94104.

Palacios Picos, A.; López-Pastor, V., \& Fraile Aranda, A. (2018). Cuestionario de percepción de competencias docentes de Educación Física / Perception Questionnaire of Teaching. Competences in Physical Education. Revista Internacional de Medicina y Ciencias de la Actividad Física y el Deporte. vol. efirst. *. Http://cdeporte.rediris.es/revista/

Zayapragassarazan, Z., \& Kumar, S. (2012). Active learning methods. Online Submission, 19(1), 3-5. 